\title{
SINTONIZAÇÃO DE ESTADOS QUÂNTICOS: UM ESTUDO NUMÉRICO DO OSCILADOR HARMÔNICO QUÂNTICO
}

\author{
Leandro de Abreu* e Alejandro López-Castillo \\ Departamento de Química, Universidade Federal de São Carlos, CP 676, 13560-970 São Carlos - SP, Brasil
}

Recebido em 28/11/11; aceito em 6/3/12; publicado na web em 2/7/12

\begin{abstract}
QUANTUM STATE TUNING: NUMERICAL STUDY OF THE QUANTUM HARMONIC OSCILLATOR. The quantum harmonic oscillator is described by the Hermite equation. ${ }^{1}$ The asymptotic solution is predominantly used to obtain its analytical solutions. Wave functions (solutions) are quadratically integrable if taken as the product of the convergent asymptotic solution (Gaussian function) and Hermite polynomial, ${ }^{1}$ whose degree provides the associated quantum number. Solving it numerically, quantization is observed when a control real variable is "tuned" to integer values. This can be interpreted by graphical reading of $\Psi(\mathrm{x})$ and $|\Psi(\mathrm{x})|^{2}$, without other mathematical analysis, and prove useful for teaching fundamentals of quantum chemistry to undergraduates.
\end{abstract}

Keywords: harmonic oscillator; numerical solution; quantization.

\section{INTRODUÇÃO}

\section{Apresentação geral}

A Equação de Schrödinger (independente do tempo) é uma equação diferencial homogênea de segunda ordem, que pode ter soluções analíticas para sistemas descritos como problemas de uma partícula efetiva (Equação1), como é o caso do oscilador harmônico quântico.

$$
\left[\nabla_{\mathrm{r}}^{2}-\frac{8 \pi \mathrm{m}}{\mathrm{h}^{2}}[\mathrm{~V}(\mathrm{r})-\mathrm{E}]\right] \Psi(\mathrm{r})=0
$$

em que $\mathbf{r}$ representa o vetor posição da partícula que compõe o problema, $\mathrm{V}(\mathbf{r})$ é o operador energia potencial do sistema, $-\frac{\mathrm{h}^{2}}{8 \pi \mathrm{m}} \nabla_{\mathrm{r}}^{2}$ é o operador energia cinética, E é a energia total do sistema, $\Psi(\mathbf{r})$ é a função de onda que descreve o sistema, h é a constante de Planck e m é a massa do sistema.

A Química Quântica, normalmente, é introduzida nos cursos de graduação em Química sem que haja um embasamento físico e matemático adequado para que a transição do modelo clássico para o modelo quântico da matéria seja feita de forma plenamente satisfatória. Desta maneira, verifica-se a existência de dificuldades de entendimento das equações e das funções de onda por parte dos alunos, mesmo para os casos em que é possível obter soluções analíticas, ou seja, em geral para os casos mais simples.

Como a Equação de Schrödinger é uma equação diferencial de $2^{\mathrm{a}}$ ordem (EDO), pode ser quebrada em duas equações diferenciais ordinárias acopladas de $1^{\mathrm{a}}$ ordem. Pela introdução de uma metodologia numérica simples e intuitiva de solução, como o método de Euler, ${ }^{2}$ o sistema de equações é reduzido a apenas uma equação de identidade e outra de recorrência para que os problemas sejam resolvidos numericamente, ou seja, são necessárias apenas duas linhas de equações. Sendo assim, tal método numérico poderia auxiliar no aprendizado da Química Quântica, podendo ser útil para a visualização e interpretação de soluções de sistemas que são solúveis analiticamente (ou não), diminuindo os empecilhos matemáticos.

De maneira geral, as equações de identidade e de recorrência que o método de Euler fornece para a resolução numérica de qualquer
EDO de $2^{\mathrm{a}}$ ordem são, respectivamente:

$$
\frac{\mathrm{w}(\mathrm{x}+\Delta \mathrm{x})-\mathrm{w}(\mathrm{x})}{\Delta \mathrm{x}}=\mathrm{u}(\mathrm{x})
$$

$\mathrm{e}$

$$
\frac{\mathrm{u}(\mathrm{x}+\Delta \mathrm{x})-\mathrm{u}(\mathrm{x})}{\Delta \mathrm{x}}=\mathrm{f}(\mathrm{x}, \mathrm{w})
$$

sendo que $f(x, w)$ representa $\frac{d^{2} w(x)}{d x^{2}}$ e $u(x)$ representa $\frac{d w(x)}{d x}$.

\section{O oscilador harmônico}

A Equação de Schrödinger de um oscilador harmônico unidimensional é dada pela Equação 4:

$$
\left[\frac{\mathrm{d}^{2}}{\mathrm{dx}^{2}}-\frac{8 \pi \mu}{\mathrm{h}^{2}}\left[\frac{1}{2} \mathrm{kx}^{2}-\mathrm{E}\right]\right] \Psi(\mathrm{x})=0
$$

sendo $\mathrm{k}$ a constante de força do oscilador associada à energia potencial do sistema $\left(\frac{1}{2} \mathrm{kx}^{2}\right)$, x é o deslocamento apresentado pelo oscilador em relação à posição de equilíbrio e $\mu$ é a massa reduzida do sistema.

A Equação 4, uma EDO de $2^{\mathrm{a}}$ ordem, pode ser escrita como duas EDO's de $1^{\mathrm{a}}$ ordem da seguinte maneira:

$$
\frac{d \Psi(x)}{d x}=u(x)
$$

$\mathrm{e}$

$$
\frac{\mathrm{du}(\mathrm{x})}{\mathrm{dx}}=\frac{8 \pi \mu}{\mathrm{h}^{2}}\left[\frac{1}{2} \mathrm{kx}^{2}-\mathrm{E}\right] \Psi(\mathrm{x})
$$

sendo que $\mathrm{u}(\mathrm{x})$ descreve a derivada de $\Psi(\mathrm{x})$.

Essas duas equações podem ser resolvidas numericamente utilizando-se derivadas finitas (método de Euler) ${ }^{2}$ dadas por $\frac{\mathrm{df}}{\mathrm{dx}} \cong \frac{[\mathrm{f}(\mathrm{x}+\Delta \mathrm{x})-\mathrm{f}(\mathrm{x})]}{\Delta \mathrm{x}}$. Dessa maneira, as Equações 5 e 6 podem ser escritas da seguinte maneira, respectivamente:

$$
\Psi(\mathrm{x}+\Delta \mathrm{x})=\Psi(\mathrm{x})+\mathrm{u}(\mathrm{x}) \Delta \mathrm{x}
$$

$$
\mathrm{u}(\mathrm{x}+\Delta \mathrm{x})=\mathrm{u}(\mathrm{x})+\frac{8 \pi \mu}{\mathrm{h}^{2}}\left[\frac{1}{2} \mathrm{kx}^{2}-\mathrm{E}\right] \Psi(\mathrm{x}) \Delta \mathrm{x},
$$


ou seja, dadas as condições iniciais $\Psi\left(\mathrm{x}_{0}\right)$ e $\mathrm{u}\left(\mathrm{x}_{0}\right)$, obtêm-se quaisquer $\Psi(\mathrm{x})$ e $\mathrm{u}(\mathrm{x})$ para $\Delta \mathrm{x}$ suficientemente pequeno, por exemplo, $\mathrm{x}_{0}=$ -10 bohr, $\Psi\left(\mathrm{x}_{0}\right)=0,001, \mathrm{u}\left(\mathrm{x}_{0}\right)=0,001$ e $\Delta \mathrm{x}=10^{-6}$ bohr. Obviamente, outras técnicas de solução numérica de equações diferenciais, como os métodos gerais Runge-Kutta, ${ }^{2}$ podem ser utilizadas.

Em princípio, no processo de integração numérica da Equação 4, a energia $E$ pode assumir qualquer valor real. Contudo, a probabilidade de se encontrar a "partícula" em todo espaço (P) deve ser sempre finita e unitária, com $\mathrm{P}$ definida da seguinte maneira:

$$
\mathrm{P}=\int_{-\infty}^{\infty}|\Psi(\mathrm{x})|^{2} \mathrm{dx}=1
$$

Sabe-se da solução analítica do oscilador harmônico que a Equação 7 é obedecida apenas se $\frac{\mathrm{E}}{\mathrm{h} v}$ for igual a um semi-inteiro positivo dado por $\left(\mathrm{n}+\frac{1}{2}\right)$, sendo $v=\frac{1}{2 \pi} \sqrt{\frac{\mathrm{k}}{\mu}}$. Neste caso, $\Psi(\mathrm{x})$ é dita quadrado integrável. ${ }^{3}$ Caso contrário, $\mathrm{P}$ diverge e $\Psi(\mathrm{x})$ não é de quadrado integrável. ${ }^{3}$ Esta condição de que n só pode assumir valores inteiros pode ser vista numericamente, sem nenhuma suposição adicional, em um processo de sintonização, que será ilustrado neste trabalho.

\section{Outros sistemas unidimensionais}

De maneira geral, a Equação 6 pode ser escrita, genericamente, para qualquer problema unidimensional da seguinte forma:

$$
\frac{\mathrm{du}(\mathrm{x})}{\mathrm{dx}}=\frac{8 \pi \mu}{\mathrm{h}^{2}}[\mathrm{~V}(\mathrm{x})-\mathrm{E}] \Psi(\mathrm{x})
$$

com $\mathrm{V}(\mathrm{x})$ sendo o termo de energia potencial que descreve o sistema a ser estudado.

A Equação 8 pode ser resolvida de maneira análoga à Equação 6, em princípio, através da seguinte generalização da Equação 6':

$$
\mathrm{u}(\mathrm{x}+\Delta \mathrm{x})=\mathrm{u}(\mathrm{x})+\frac{8 \pi \mu}{\mathrm{h}^{2}}[\mathrm{~V}(\mathrm{x})-\mathrm{E}] \Psi(\mathrm{x}) \Delta \mathrm{x}
$$

Alguns exemplos de sistemas unidimensionais usuais em Química Quântica são:

- oscilador anarmônico: $\mathrm{V}(\mathrm{x})=\frac{1}{2} \mathrm{kx}^{2}+\sum_{\mathrm{i}=1}^{\mathrm{N}} \gamma_{\mathrm{i}} \mathrm{x}^{\mathrm{i}+2}$, com $\mathrm{N}$ sendo o número de termos anarmônicos adicionados ao potencial harmônico e $\gamma_{i}$ as constantes de anarmonicidade associadas a cada termo de correção. É frequentemente aplicado à descrição de vibrações em moléculas diatômicas;

oscilador de Morse: ${ }^{4} \mathrm{~V}(\mathrm{x})=\mathrm{D}\left[1-\mathrm{e}^{-\mathrm{ax}}\right]^{2}$, sendo que $\mathrm{D}$ representa a energia de dissociação do oscilador e a está relacionada à largura do poço de energia potencial. Esse modelo é frequentemente aplicado à descrição de vibrações em moléculas diatômicas; barreiras de inversão ${ }^{5}$ e de rotação: $\mathrm{V}(\mathrm{x})=\sum_{\mathrm{i}=0}^{\mathrm{N}} \gamma_{\mathrm{i}} \mathrm{x}^{2 \mathrm{i}}$, com x representando ângulo de distorção relativo ao ângulo de equilíbrio $\mathrm{x}_{0}$, $\mathrm{N}$ sendo o número de termos escolhidos para a descrição do poço de energia potencial e $\gamma_{\mathrm{i}}$ os pesos de cada termo utilizado. Pode ser utilizado na descrição da barreira de inversão da molécula como $\mathrm{NH}_{3}$ e da rotação interna entre os hidrogênios de moléculas como $\mathrm{H}_{2} \mathrm{O}_{2}$.

Outros modelos bi e tridimensionais também poderiam ser estudados utilizando o método de Euler.

\section{METODOLOGIA}

Neste trabalho foram utilizadas unidades atômicas $\left(\frac{h}{2 \pi}=1\right.$ u.a.' $\mathrm{m}_{\mathrm{e}}=1$ u.a. - massa do elétron $-\mathrm{e} \mathrm{q}_{\mathrm{e}}=1$ u.a. - carga elementar) $\mathrm{e}$ valores de $\mathrm{k} \mathrm{e} \mu$ referentes ao estado fundamental da molécula $\mathrm{H}_{2}$ ( $\mathrm{k}=0,3692872$ u.a. $^{6}$ e $\mu=918,3076335$ u.a.). Os cálculos foram realizados para os números (reais) $\mathrm{s}=[-0,01 ; 0 ; 0,01 ; 0,99 ; 1 ; 1,01$; $1,99 ; 2 ; 2,01]$, sendo que $\mathrm{s}=\mathrm{n}$ para números inteiros. As condições iniciais utilizadas foram $\mathrm{x}_{0}=0,0$; ou seja, a posição de equilíbrio, embora $\mathrm{x}_{0}$ possa ter qualquer valor, $\Psi\left(\mathrm{x}_{0}\right)=1,0$ e $\mathrm{u}\left(\mathrm{x}_{0}\right)=0,0$; exceto para os cálculos realizados com $\mathrm{s}=[0,99 ; 1,0 ; 1,01]$ em que foram utilizadas $\Psi\left(\mathrm{x}_{0}\right)=0,0$ e $\mathrm{u}\left(\mathrm{x}_{0}\right)=1,0$. Foram calculados 500.000 pontos para deslocamentos positivos e 500.000 pontos para deslocamentos negativos, sendo $\Delta \mathrm{x}=2 \cdot 10^{-6} \mathrm{bohr}$. As funções de onda foram normalizadas durante o processo de cálculo numérico pelo uso do método de integração numérica dos trapézios, ${ }^{2}$ considerando que:

$$
\int_{-\infty}^{\infty}|\Psi(\mathrm{x})|^{2} \mathrm{dx}=\frac{1}{2} \sum_{\mathrm{i}=1}^{\mathrm{N}-1}\left[\left|\Psi\left(\mathrm{x}_{\mathrm{i}}\right)\right|^{2}+\left|\Psi\left(\mathrm{x}_{\mathrm{i}+1}\right)\right|^{2}\right]
$$

sendo $\mathrm{N}$ o número de pontos. Além disso, também foram calculadas as densidades de probabilidade $|\Psi(\mathrm{x})|^{2}$ e a energia potencial $\mathrm{V}(\mathrm{x})=\frac{1}{2} \mathrm{kx}^{2}$.

\section{RESULTADOS E DISCUSSÃO}

Nas Figuras 1, 2 e 1S (material suplementar) são apresentadas curvas referentes à $\Psi(\mathrm{x})$ (normalizada) e $|\Psi(\mathrm{x})|^{2}$ obtidas numericamente para cada s estudado, além da curva calculada $\mathrm{V}(\mathrm{x})$ (curvas ponto-tracejadas) e as energias totais calculadas para cada valor de $\mathrm{s}$ (retas pontilhadas).

Observa-se nas Figuras 1, 2 e 1S (material suplementar) que $\Psi(\mathrm{x})$ e $|\Psi(\mathrm{x})|^{2}$ divergem quando $|\mathrm{x}| \rightarrow \infty$, exceto para casos em que $\mathrm{o}$ valor de $\mathrm{s}$ é inteiro (Figuras $1 \mathrm{~b}, 2 \mathrm{~b}$ e $1 \mathrm{Sb}$ ). Para valores inteiros de s verifica-se que $\Psi(\mathrm{x})$ e $|\Psi(\mathrm{x})|^{2}$ são finitas em todo o domínio em que estão definidas e convergem para 0 quando $|\mathrm{x}| \rightarrow \infty$. $\Psi(\mathrm{x})$ é uma função par $\left(\frac{\mathrm{d} \Psi(0)}{\mathrm{dx}}=0\right)$ se s é par e $\Psi(\mathrm{x})$ é ímpar $(\Psi(0)=0)$ se s for ímpar. Além disso, observa-se numericamente um padrão de comportamento para o número de nós que $\Psi(\mathrm{x})$ apresenta em função de $\mathrm{s}$, quando s é inteiro. O número de nós, nestes casos, é sempre igual a s. Pode-se verificar ainda o efeito de tunelamento, além dos limites clássicos de deslocamentos definidos pelo poço de energia potencial para uma dada energia total.

A quantização observada na natureza está sempre relacionada a um fenômeno de confinamento espacial dos sistemas. De maneira geral, qualquer sistema que seja caracterizado por um poço de energia potencial confinante apresentará quantização. $\mathrm{O}$ oscilador harmônico unidimensional é um modelo que representa sistemas confinados espacialmente por um poço de energia potencial do tipo $\mathrm{V}(\mathrm{x})=\frac{1}{2} \mathrm{kx}^{2}$, como observado nas Figuras 1, 2 e 1S.

Um sistema confinado, segundo a teoria de De Broglie, irá apresentar interferência de onda de matéria. Para o caso em que s é inteiro (Figuras 1b, 2b e 1Sb), observam-se (numericamente) interferências construtivas, permitindo o aparecimento de estados quânticos. Para o caso em que s não é inteiro (Figuras $1 \mathrm{a}, 1 \mathrm{c}, 2 \mathrm{a}, 2 \mathrm{c}, 1 \mathrm{Sa}$ e 1Sc), observam-se interferências destrutivas e a densidade de probabilidade $|\Psi(\mathrm{x})|^{2}$ será, relativamente, nula para a região confinada e divergirá fora dessa região.

A Figura 3 ilustra, para o caso do oscilador harmônico quântico, que a divergência de $\Psi(\mathrm{x})$ se torna menos acentuada conforme s $\rightarrow 2$ para os seguintes valores $s=[1,99 ; 1,999 ; 2 ; 2,001 ; 2,01]$. Além disso, nas Figuras 1, 2 e 1S, verifica-se que os sinais da divergência de $\Psi(\mathrm{x})$ para $\mathrm{s}<n$ e $\mathrm{s}>n$ são invertidos (para as mesmas condições iniciais). Portanto, é possível encontrar os autovalores, previamente desconhecidos, de um sistema genérico a partir de uma varredura 
a) $s=-0,01$
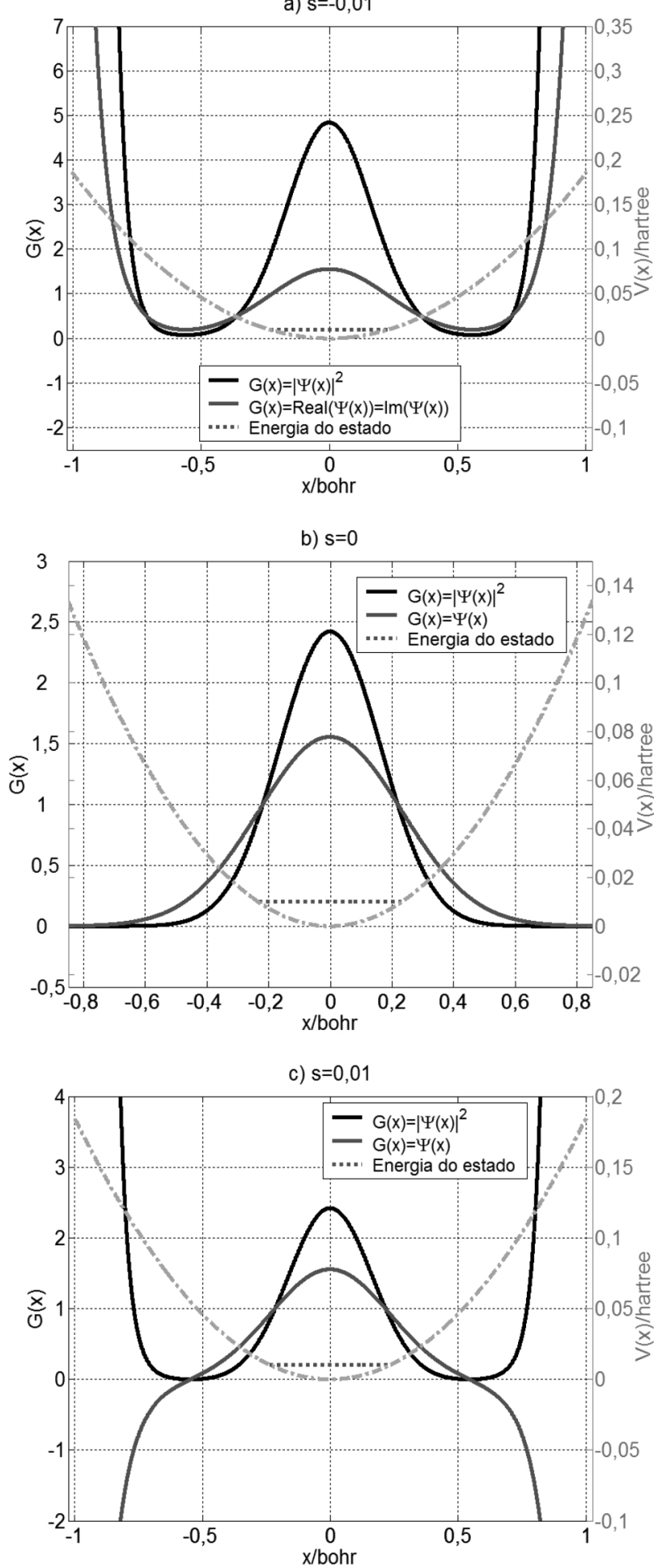

Figura 1. $\Psi(x)$ (normalizada) $e|\Psi(x)|^{2}$ para $\left.\left.a\right) s=-0,01, b\right) s=0$ e c) $s=$ 0,$01 ; V(x)$ (curva ponto-tracejada) e E (energia total) para cada valor de $s$ (reta pontilhada)

sobre os possíveis valores que E possa assumir e da observação da convergência de $\Psi(\mathrm{x})$ no momento que os sinais invertem, isto é, realizando o processo de sintonização.

Em geral, a sintonização (quântica) pode ser realizada para qualquer sistema de qualquer dimensão utilizando qualquer método numérico. Em particular, apresentamos a sintonização do oscilador harmônico unidimensional utilizando o método de Euler.
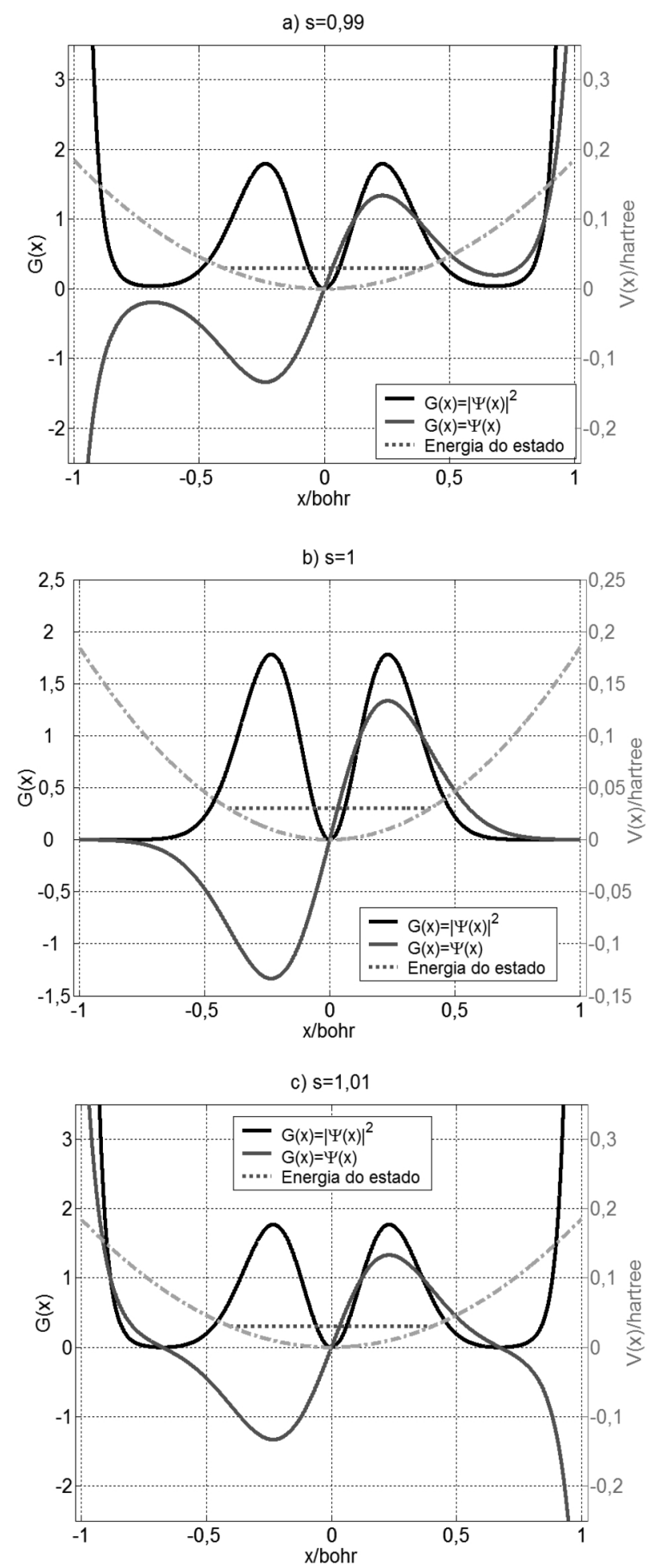

Figura 2. $\Psi(x)$ (normalizada) $e|\Psi(x)|^{2}$ para a) $\left.s=0,99, b\right) s=1$ e c $) s=$ 1,01; V(x) (curva ponto-tracejada) e E para cada valor de s (reta pontilhada)

\section{CONCLUSÃO}

O uso do cálculo numérico na construção de funções de onda de possíveis estados quânticos e de argumentos intuitivos e simples permite interpretar o surgimento, o significado da quantização e as propriedades que emergem com a quantização, através da simples leitura dos gráficos que representam $\Psi(\mathrm{x}) \mathrm{e}|\Psi(\mathrm{x})|^{2}$, sem a necessidade 


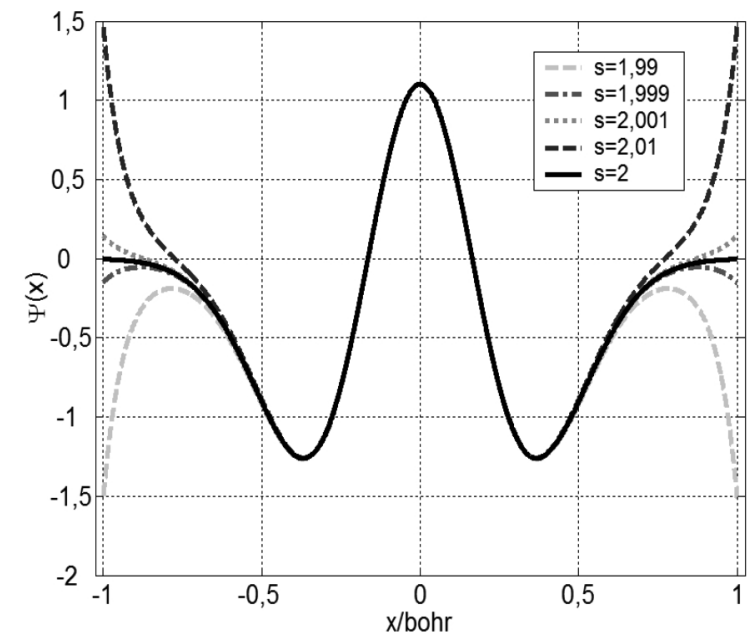

Figura 3. Convergência de $\Psi(x)$ para $s \rightarrow 2$

da análise de equações e funções matemáticas. Isto pode ser útil para facilitar o aprendizado dos fundamentos da Química Quântica em nível de graduação.

A análise dos resultados deste trabalho permite observar a "sintonização" da quantização quando a variável s se aproxima de um inteiro, isto é, quando ela se torna um número quântico.

\section{MATERIAL SUPLEMENTAR}

O material suplementar apresenta as curvas referentes à $\Psi(\mathrm{x})$ (normalizada), $|\Psi(\mathrm{x})|^{2}, \mathrm{~V}(\mathrm{x})$ e as energias totais calculadas para $\mathrm{s}=$ $[1,99 ; 2 ; 2,01]$, de forma similar às Figuras 1 e 2 . Está disponível em http://quimicanova.sbq.org.br, na forma de arquivo PDF e com acesso livre.

\section{REFERÊNCIAS}

1. Abramowitz, M.; Stegun, I. A.; Handbook of mathematical functions with formulas, graphs, and mathematical tables, $10^{\text {th }}$ ed., John Wiley and Sons: New York, 1972.

2. Hoffman, J. D.; Numerical methods for engineers and scientists, $2^{\text {nd }}$ ed., Marcel Dekker Inc.: New York, 2001.

3. Levine, I. N.; Quantum Chemistry, $5^{\text {th }}$ ed., Prentice Hall: Upper Saddle River, 2000.

4. Morse, P. M.; Phys. Rev. 1929, 34, 57.

5. Swalen, J. D.; Ibers, J. A.; J. Chem. Phys. 1962, 36, 1914.

6. Irikura, K. K.; J. Phys. Chem. Ref. Data 1990, 36, 389. 


\section{SINTONIZAÇÃO DE ESTADOS QUÂNTICOS: UM ESTUDO NUMÉRICO DO OSCILADOR HARMÔNICO QUÂNTICO}

Leandro de Abreu* e Alejandro López-Castillo

Departamento de Química, Universidade Federal de São Carlos, CP 676, 13560-970 São Carlos - SP, Brasil

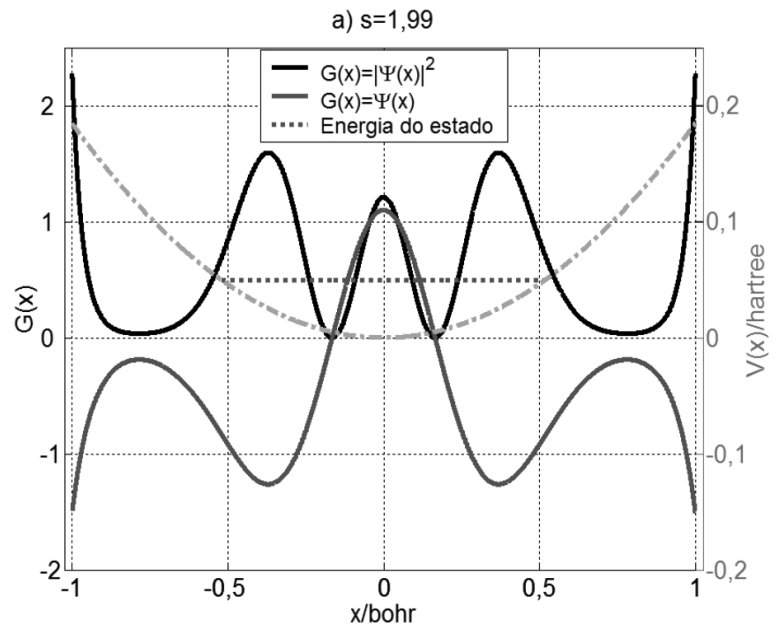

b) $s=2$
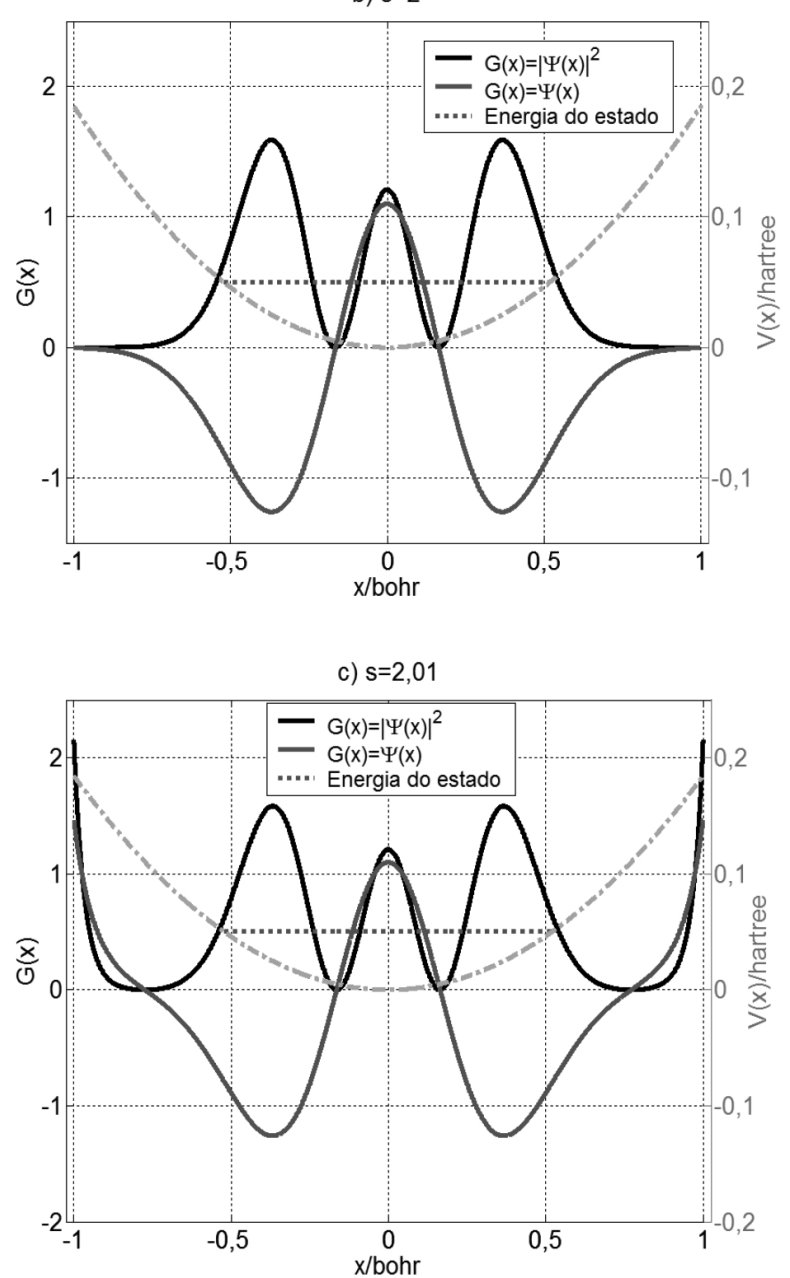

Figura 1S. $\Psi(x)$ (normalizada) e $|\Psi(x)|^{2}$ para $\left.\left.a\right) s=1,99 ; b\right) s=2$ e c) $s=2,01 ; V(x)$ (curva ponto-tracejada) e E para cada valor de $s$ (reta pontilhada) 